\title{
Application of passive wedge absorbers for improving the performance of precision-science experiments
}

\author{
Diktys Stratakis \\ Fermi National Accelerator Laboratory, Batavia, Illinois 60510, USA
}

(Received 13 November 2018; published 22 May 2019)

\begin{abstract}
A scheme is discussed for momentum selection and momentum-spread reduction for muon-based experiments. The concept relies on placing a wedge absorber at a point along a beam transport system with nonzero dispersion. The technique has direct relevance to precision-science experiments such as the Fermilab Muon g-2 Experiment as it can enhance the muon beam intensity and therefore minimize the statistical uncertainty of the anomalous magnetic moment measurement. This paper presents a theoretical and numerical study about the orientation, material, geometrical parameters, and performance of this wedge. Results suggest a considerable increase in muon intensity for the Muon g-2 Experiment, when the optimal wedge is introduced along the beam path.
\end{abstract}

DOI: 10.1103/PhysRevAccelBeams.22.053501

\section{INTRODUCTION}

The Fermilab Muon g-2 Experiment [1] signals the beginning of an important research program that uses muons as a probe to study physics beyond the Standard Model. The ultimate goal of the Muon g-2 Experiment is to measure the anomalous magnetic moment of the muon with an unprecedented precision of $0.14 \mathrm{ppm}$ [2]. For the experiment, a polarized muon beam is formed by capturing forward muons from pion decay in flight. The muons then are injected into a storage ring with a uniform vertical magnetic field. The magnetic moment of the muon causes it to rotate or precess around the central axis of the magnetic field. Measurements of this precession frequency and the strength of the magnetic field are used to extract the anomalous magnetic moment of the muon [3].

The Muon g-2 Experiment will transport muons around a $7.11 \mathrm{~m}$ in radius ring and the polarization of their decay products will be observed [4]. Since the uncertainty of the measurement is largely dependent on statistics, it is essential to place as many muons as possible into a stable orbit in the ring. It is important to emphasize that the storage ring has a very narrow momentum acceptance, meaning that only particles within $\sigma / p_{m}=0.12 \%$, where $\sigma$ is the rms momentum spread and $p_{m}$ is the mean momentum, will eventually survive. In contrast, the line that delivers beam to the ring has a larger acceptance, i.e.,

Published by the American Physical Society under the terms of the Creative Commons Attribution 4.0 International license. Further distribution of this work must maintain attribution to the author(s) and the published article's title, journal citation, and DOI. $\sigma / p_{m}=1.26 \%$. Results from numerical simulations [5,6], displayed in Fig. 1, predict that only $10 \%$ of the delivered muons have a momentum that is within $0.12 \%$ of $p_{m}$. Hence, it is likely that the performance of the Muon $g-2$ Experiment will be limited by the momentum acceptance. For this reason, it is advantageous to develop new techniques that could reduce the momentum spread of the incoming beam and therefore increase the number of stored muons.

In this paper we present a scheme to increase the beam intensity, consequently improving the performance of the

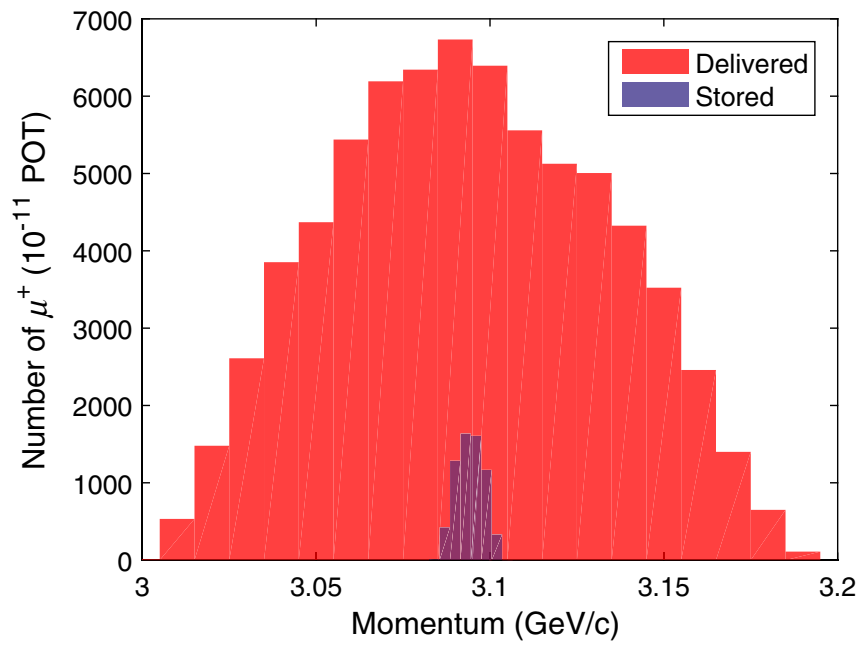

FIG. 1. Momentum distribution of the Muon Campus beam at the entrance of the storage ring (red). Momentum distribution of the same beam but after completing 100 turns inside the ring (blue). Notice that the rms momentum spread of the surviving beam is $0.12 \%$ meaning that a big fraction of the incoming beam is lost. POT refers to the protons on target. 
Muon g-2 Experiment. The key element of our scheme is to introduce emittance exchange to the incoming muon beam $[7,8]$. With this technique, a dispersive beam is passed through a wedge in such a way that the high-energy particles traverse more material than the low-energy ones. Essentially, the wedge creates a correlation between the amounts of energy loss within the wedge material with the energy of the individual particles when placed in a region of dispersion. As we will show, with appropriately chosen material and wedge geometry, the overall spread in momentum can be reduced. A similar concept has been previously applied [9-13] in ionization cooling channels for muon accelerators wherein a notable reduction of the $6 \mathrm{D}$ beam emittance by at least 5 orders of magnitude has been achieved in simulation.
In this study we investigate a possible configuration of a wedge system along the Fermilab Muon Campus [14,15], the facility that produces and delivers a muon beam to the Muon g-2 Experiment. We show that with emittance exchange the resulting momentum spread of the postwedge beam is not only lower than conventional designs but also maintains its value through the passage into the ring, resulting in a considerable increase of stored muons. Our findings indicate that even with the inclusion of a wedge, the muon polarization is not degraded, meaning that it remained $>90 \%$ as desired by the Muon g-2 Experiment. With the aid of this work the absorber material, thickness, and geometry are specified to a level that a practicality study can be implemented. For instance, we show that two polyethylene wedges with a longitudinal thickness of
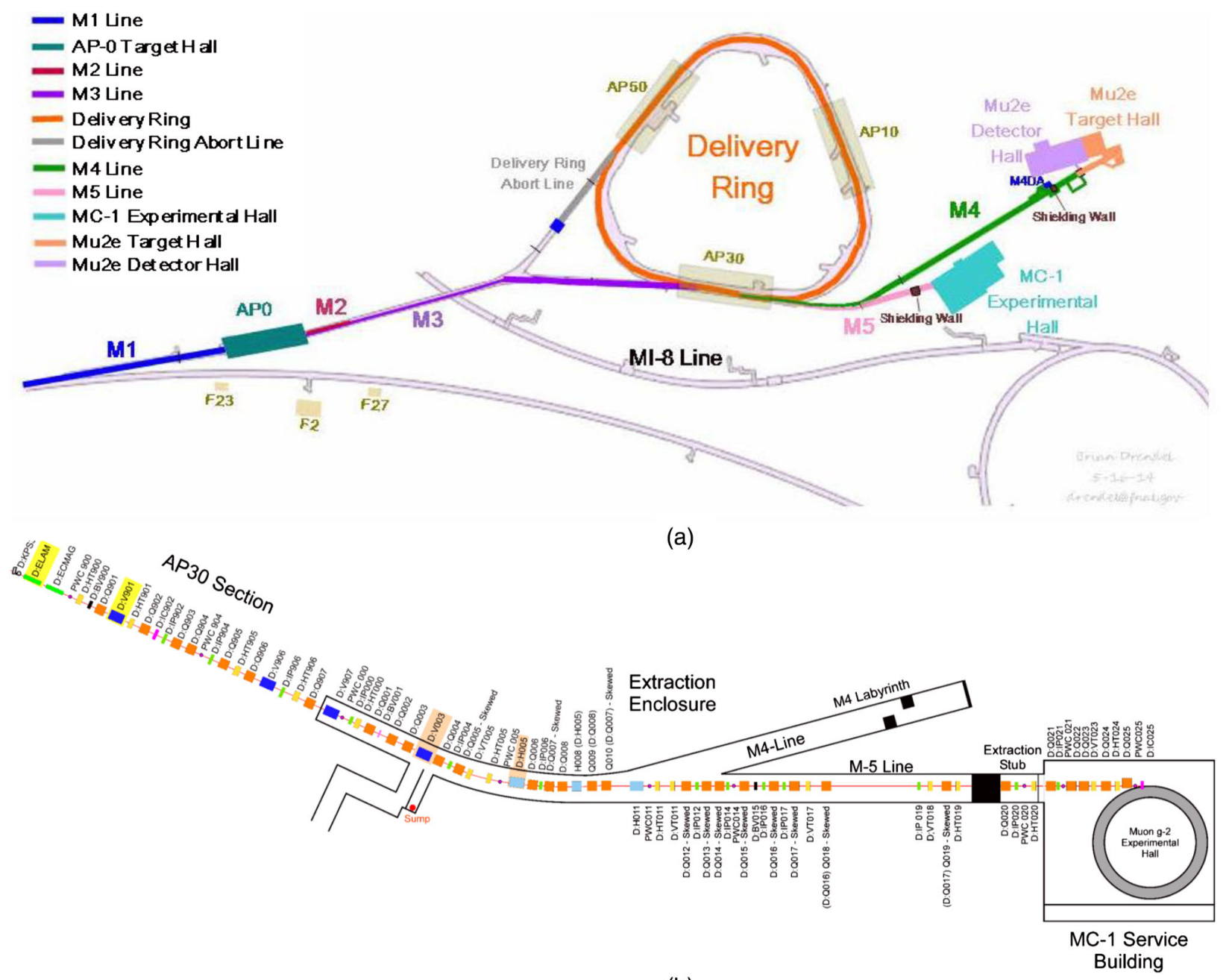

(b)

FIG. 2. (a) Schematic representation of the Muon Campus accelerator complex that is used by the Muon g-2 Experiment. Secondaries are produced on a target that then travel through the M2 and M3 lines, which are designed to capture as many $3.1 \mathrm{GeV} / \mathrm{c}$ muons from pion decays as possible. The beam is injected into the DR wherein a kicker is used to remove the protons, the resulting muon beam is then extracted into the M4 line, and the muon beam is eventually transferred to the M5 line that leads to the muon storage ring (enclosed in the MC1 hall). The combined M2 and M3 line and M4 and M5 line lengths are 280 and $128 \mathrm{~m}$, respectively, along with the DR that has a circumference of $505 \mathrm{~m}$. (b) Schematic layout of the M4-M5 lines. Note that ECMAG is a bending magnet and its upstream edge signals the start of the M4 line $(S=0 \mathrm{~m})$. AP designates antiproton. 
$125 \mathrm{~mm}$ with a tapered edge leading to a point near the beam center are sufficient to achieve a considerable increase in beam intensity.

The outline of the paper is as follows. In Sec. II we overview the Muon Campus beam lines. In Sec. III, we discuss the principle of emittance exchange and how it can be implemented at the Muon Campus. We also specify the optimum material, angle, and thickness of the wedge. In Sec. IV we report the results from our preliminary simulations modeling the aforementioned system. In the last part of the paper (Sec. V) we present our conclusions.

\section{BEAM LINE DESCRIPTION}

Figure 2(a) displays a schematic layout of the Fermilab Muon Campus, where $8 \mathrm{GeV}$ protons are transported via the M1-line to an Inconel target [16] for the Muon g-2 Experiment. A secondary beam from the target is collected using a lithium lens, where the positively charged particles with $3.1 \mathrm{GeV} / \mathrm{c}( \pm 10 \%)$ are selected by using a bending magnet. The secondary beam leaves the target station and travels through the M2 and M3 line, which is designed to capture as many $3.1 \mathrm{GeV} / \mathrm{c}$ muons as possible from the pion decays. The beam is then injected into the delivery ring (DR), where the majority of pions decay into muons after several revolutions. The DR is also used to separate muons in time from the heavier protons. A kicker is then used to remove the protons, where the muon beam is extracted into the M4 line.

Injection from the M3 line and extraction to the M4 line takes place in the same straight section with the latter happening in the downstream half. Figure 2(b) displays a schematic layout of the $128.0 \mathrm{~m}$ long M4-M5 lines that transfer the beam from the DR towards the storage ring of the Muon g-2 Experiment while Fig. 3(a) shows the corresponding baseline design beta and dispersion functions along these lines [17]. The M5 line includes a $27.1^{\circ}$ horizontal bend string at $S \approx 46.0 \mathrm{~m}$ that provides the proper entry position and angle into the storage ring, and right before the end of the M5 line there is a strong focusing and tunable final focus section, using four quadrupole magnets at $S=119.8 \mathrm{~m}$, which provide optical matching to the storage ring.

The radius of the storage ring [18] of the Muon g-2 Experiment is $7.11 \mathrm{~m}$ with its field designed to be vertical and uniform at a central value of $1.45 \mathrm{~T}$. Electrostatic quadrupoles are used for vertical focusing of the beam. Muons are injected into the ring through the narrow horizontal restrictions, i.e., $18 \mathrm{~mm}$, of a superconducting $1.7 \mathrm{~m}$ long inflector $[19,20]$ which bucks the main dipole field so that the beam transverses the inflector without significant deflection and exits on a trajectory tangent to a displaced circular orbit. One quarter of the way around the ring, a magnetic kicker applies an outward transverse $\sim 10$ mrad angular kick during the first turn only, aligning the muon beam on the desired central orbit. Finally, five

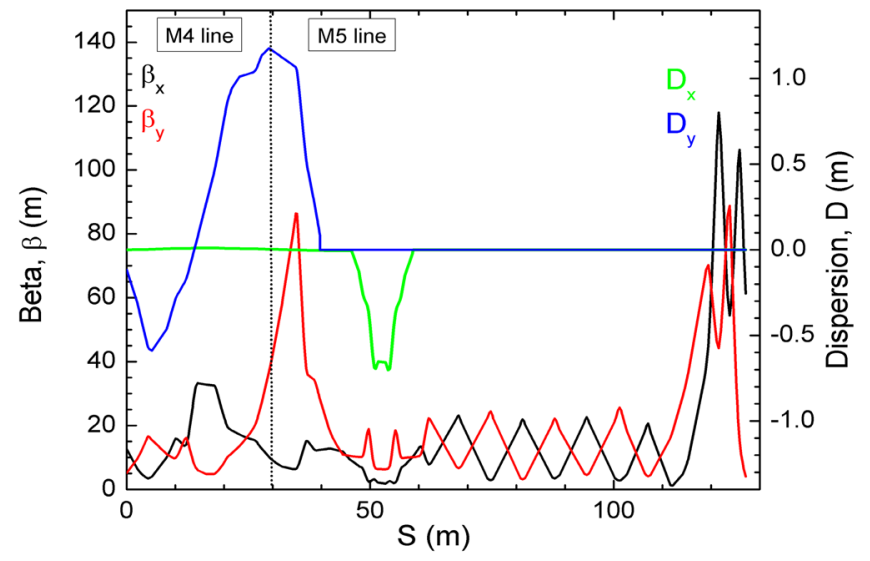

(a)

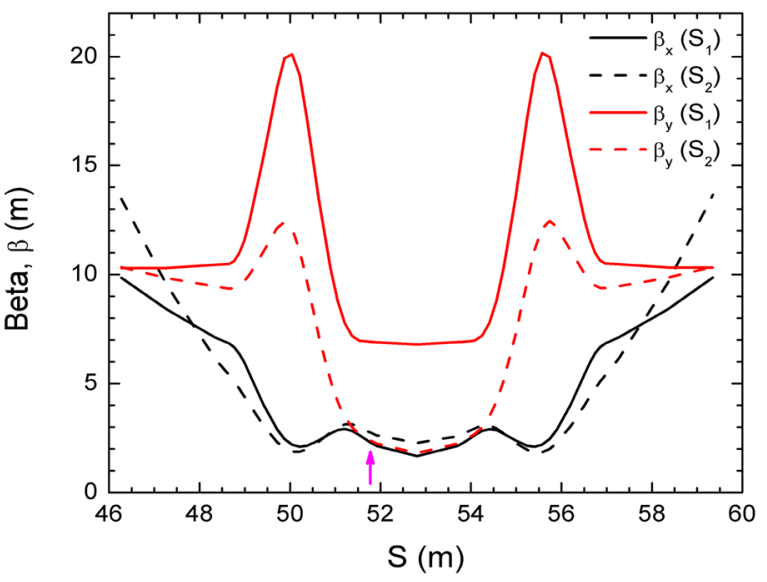

(b)

FIG. 3. (a) Baseline design optics functions for the M4 and M5 lines where the M5 ends at the entrance of the muon storage ring. The M5 starts at $S \approx 30.0 \mathrm{~m}$ and a $27.1^{\circ}$ horizontal bend string begins at $S \approx 46.0 \mathrm{~m}$ that provides proper entry position into the ring. (b) Closeup along the $\sim 13 \mathrm{~m}$ long M5 horizontal bend string. The solid curves show the optical functions of the baseline design which we call solution $S_{1}$. The dashed curves show an alternative scheme that delivers the same number of muons as the baseline but with a suppressed vertical beta function along the bend area and therefore is expected to reduce the scattering effect. We call this solution $S_{2}$. Note that both solutions have almost equivalent dispersion along the string.

collimators are placed around the ring which also limit its aperture to a circle with a $45 \mathrm{~mm}$ radius.

\section{DESIGN CONSIDERATIONS}

\section{A. Emittance exchange}

In order to reduce the momentum spread of the muon beam it must be arranged that the high-energy particles lose more energy than the low-energy ones, thus forcing the distribution toward a smaller energy spread, at the expense of an average energy loss. This process can be enhanced by placing a wedge-shaped absorber in a lattice location where there is dispersion and tailoring the wedge so as to absorb 
more energy from the higher-momentum muons and less from the lower-momentum ones. Such attempt could potentially increase the rate of storable muons.

Besides the desired ionization energy loss, the passage of muons through material will cause trajectories to be scattered due to multiple scattering interactions, hence creating transverse emittance growth. If the beam grows transversely, it can degrade the capture efficiency since the overall acceptance of the storage ring is defined by its geometry and focusing parameters. The equation describing the transverse emittance change as the beam passes through a material is given by $[7,21,22]$

$$
\frac{d \varepsilon_{n}}{d s}=-\frac{1}{\beta^{2}} \frac{d E_{\mu}}{d s} \frac{\varepsilon_{n}}{E_{\mu}}+\frac{1}{\beta^{3}} \frac{\beta_{T} E_{s}^{2}}{2 E_{\mu} m_{\mu} c^{2} L_{R}}
$$

where $\varepsilon_{n}$ is the normalized rms transverse emittance, $E_{\mu}$ is the muon energy in $\mathrm{GeV}, m_{\mu}$ is the muon mass, $\beta_{T}$ is the transverse betatron function within a discrete absorber, $\beta$ is the particle velocity, $c$ is the speed of light, $d E_{\mu} / d s$ is the energy loss per unit length, $L_{R}$ is the radiation length of the material, and $E_{s}$ is the characteristic scattering energy $(\sim 13.6 \mathrm{MeV})$. One can see from Eq. (1) that the degree to which the scattering of trajectories will increase the emittance of the beam depends on the material properties and the local optics at the wedge. It is expected that if the appropriate material is used and the transverse beam size is made small at the absorber, transverse emittance growth may be minimized and, in this case, one may be able to succeed in creating a beam with a slightly higher transverse emittance but with a much-reduced momentum spread.

\section{B. Choice of material}

While several materials are commercially available for constructing a wedge only a few are suitable for emittance exchange. In order to illustrate this, we establish a merit factor $Q$. Namely, if we assume that the "cooling" term is governed by $d E_{\mu} / d s$ and the "heating" term is dominated by $1 / L_{R}$, we can rank materials based on the product $Q=d E_{\mu} / d s \times L_{R}$. Our findings are displayed in Fig. 4 . While low- $Z$ materials, such as $\mathrm{Li}$ or Be achieve the highest possible $Q$, they are also attached to several safety constraints. On the other hand, commonly used materials such as $\mathrm{Al}, \mathrm{Ni}$, or $\mathrm{Fe}$ have much lower $Q$, implying worse performance. One notable material is polyethylene $\left(\mathrm{C}_{2} \mathrm{H}_{4}\right)_{n}$, which besides its relatively good properties for emittance exchange it is also cost effective, safe to operate, and can be machined easily into the desired shapes. For this reason, we consider $\left(\mathrm{C}_{2} \mathrm{H}_{4}\right)_{n}$ as the material of choice for all our subsequent studies.

\section{Choice of location}

For practical reasons, it is highly desirable to build the system without moving or adding any magnets along the

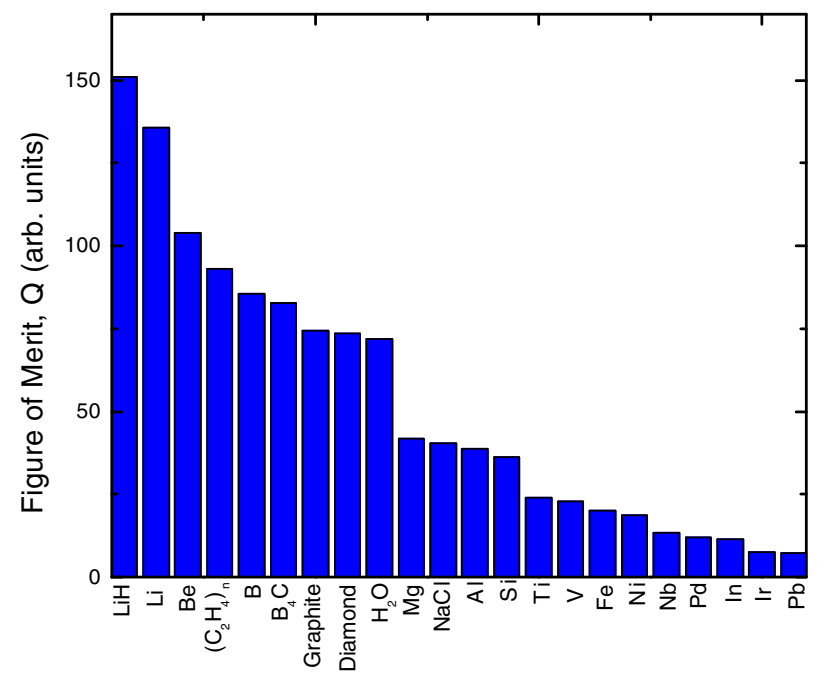

FIG. 4. Ranking of different materials based on their expected performance. Our figure of merit $Q$ is the ratio between the ionization term $d E_{\mu} / d s$ and the scattering term $1 / L_{R}$. Clearly, higher $Q$ implies better performance.

Muon Campus. On the other hand, the absorber is expected to trigger emittance growth and mismatches which, if not properly controlled, can lead to beam losses. This imposes several placement constraints since the Muon Campus has a series of tight bending and quadrupole sections, areas with elevation changes, as well as complicated injection and extraction schemes with closely packed instrumentation. Furthermore, the DR injection and DR extraction sections contain the narrowest apertures along all beam lines and therefore any upstream beam growth can significantly harm performance [5]. For this reason, we choose to place the wedge downstream of the extraction region, along the last horizontal bend string of the M5 line [see Fig. 3]. A closeup of this bend string is shown in Fig. 3(b) wherein the vertical arrow is pointing to the wedge location. There are two additional advantages of selecting this region: First, the beam is free of protons and the remaining muons have a relatively low intensity $\left(\sim 10^{5}\right.$ muons for each incoming pulse), hence energy deposition is limited to negligible levels. Second, the baseline optics design $\left[S_{1}\right.$ in Fig 3(b)] provides considerable dispersion along the M5 horizontal bend string, i.e., $D_{x}=-0.65 \mathrm{~m}$ while it simultaneously maintains a relatively small horizontal beta function $\beta_{x}=2.3 \mathrm{~m}$. One point of concern is the vertical beta function, which has a minimum of $\beta_{y}=6.9 \mathrm{~m}$. For this reason, we also consider an alternative optics scheme $\left[S_{2}\right.$ in Fig. 3(b)] that has almost the same $D_{x}$ but a minimum $\beta_{y}=2.4 \mathrm{~m}$ which is significantly lower compared to the baseline. While the required magnet strengths for $S_{1}$ and $S_{2}$ are different, both solutions are inherently designed in order to provide a beam with the same Twiss parameters at the end of the M5 line. The wedges will be placed at the same location, in both solutions, without any relocation of existing beam line components. 


\section{Choice of length and angle}

To ascertain the scale of the emittance exchange effects, we have incorporated a fast Monte Carlo [23] using a MATLAB [24] programming environment. More specifically, a high-statistics run was carried out to investigate the emittance exchange efficiency as a function of the wedge angle and its maximum thickness. We assume that both dispersion and beta functions at the absorber match the ones from $S_{1}$, namely $D_{x}=-0.65 \mathrm{~m}, \beta_{x}=2.3 \mathrm{~m}$, and $\beta_{y}=6.9 \mathrm{~m}$. We use a beam with a rms unnormalized emittance of $12.0 \mu \mathrm{m}$ in both planes which is not far from our previous measurements [14,25]. Our results for $\left(\mathrm{C}_{2} \mathrm{H}_{4}\right)_{n}$ are illustrated in Fig. 5 wherein the vertical scale on the right shows the percentage of stored muons relevant to the case without a wedge. We found that the performance was strongly correlated to the absorber angle $\theta$. For instance, for $\left(\mathrm{C}_{2} \mathrm{H}_{4}\right)_{n}$ the optimum wedge angle was found to be between 160 and $170^{\circ}$. Notice that there is a sharp increase in effectiveness with thickness which then smooths off such that the number of muons becomes independent after a particular point. This point was highly dependent on material density. For instance, for dense materials such as $\mathrm{B}_{4} \mathrm{C}\left(\rho=2.52 \mathrm{gr} / \mathrm{cm}^{3}\right)$ a thickness of $150 \mathrm{~mm}$ is adequate, while for less dense materials such as $\left(\mathrm{C}_{2} \mathrm{H}_{4}\right)_{n}\left(\rho=0.89 \mathrm{gr} / \mathrm{cm}^{3}\right)$ a wedge with a thickness of at least $220 \mathrm{~mm}$ is required.

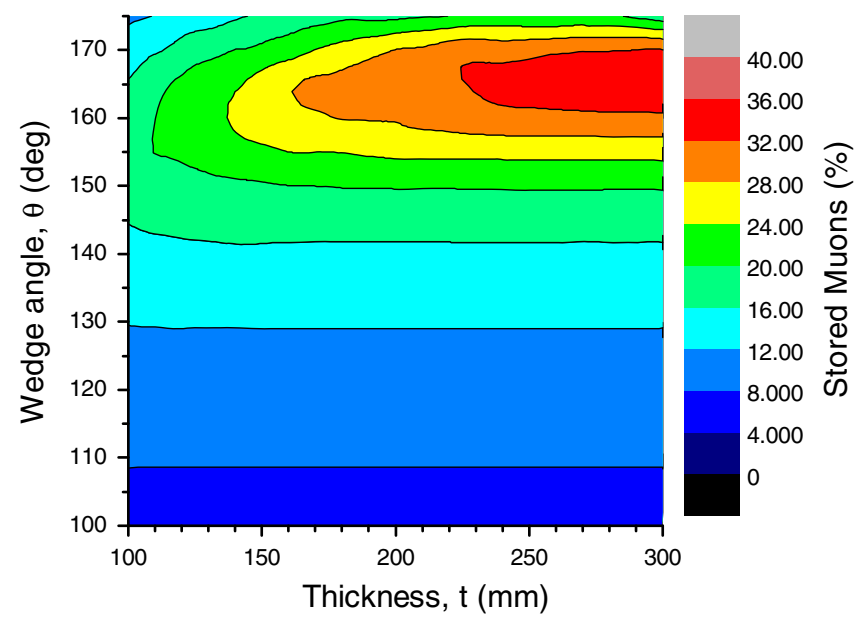

FIG. 5. Color map showing the improvement in performance as a function of thickness and angle of the wedge. We assume a $\left(\mathrm{C}_{2} \mathrm{H}_{4}\right)_{n}$ house-shaped wedge and as thickness we define the maximum distance along the beam direction. The vertical scale on the right shows the percentage of gained or lost stored muons compared to the no absorber case. The number of stored muons is increased by more than $30 \%$ when the wedge thickness is $>220 \mathrm{~mm}$ and its angle is between $160^{\circ}$ and $170^{\circ}$. The wedge is pointing along the horizontal direction with its apex placed near the beam center so that it covers at least half of the beam, mainly the high-momentum particles.

\section{E. Choice of optics}

Next, we examine the improvement in emittance exchange as a function of the horizontal lattice functions at the wedge location. For consistency with the two optics designs in Fig. 3(b), we keep $\beta_{y}$ fixed with values either $6.9 \mathrm{~m}$ [Fig. 6(a)] or $2.4 \mathrm{~m}$ [Fig. 6(b)] during our scans. Similar to Fig. 5, the vertical scale on the right is the percentage of stored muons relevant to the case without a wedge. We use a $\left(\mathrm{C}_{2} \mathrm{H}_{4}\right)_{n} 162^{\circ}$ house-shaped wedge pointing along the horizontal direction with a maximum thickness of $250 \mathrm{~mm}$.

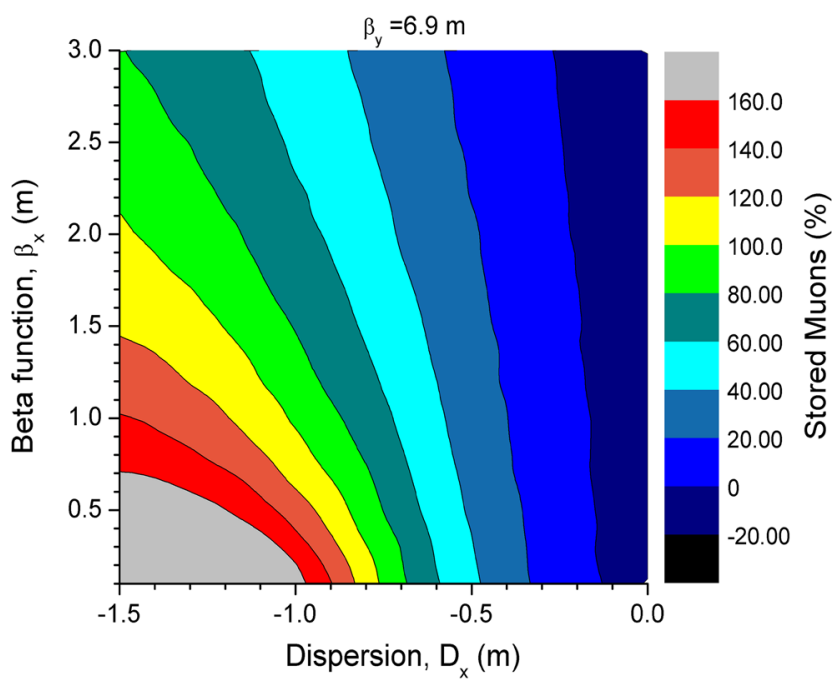

(a)

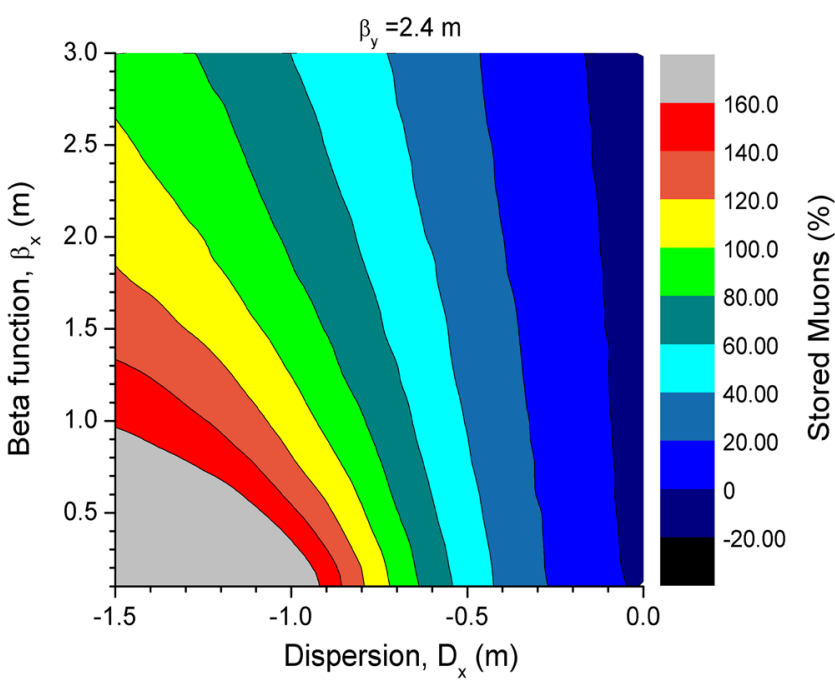

(b)

FIG. 6. Color map showing the improvement in performance as a function of horizontal lattice functions at the wedge location. The vertical scale on the right shows the percentage of gained or lost stored muons compared to the no absorber case. All studies assume a single $\left(\mathrm{C}_{2} \mathrm{H}_{4}\right)_{n} 250 \mathrm{~mm}$ thick house-shaped wedge at a $162^{\circ}$ angle. (a) Performance for the baseline lattice $S_{1}$. (b) Performance for the modified lattice $S_{2}$. The wedge is pointing along the horizontal direction with its apex placed near the beam center. 


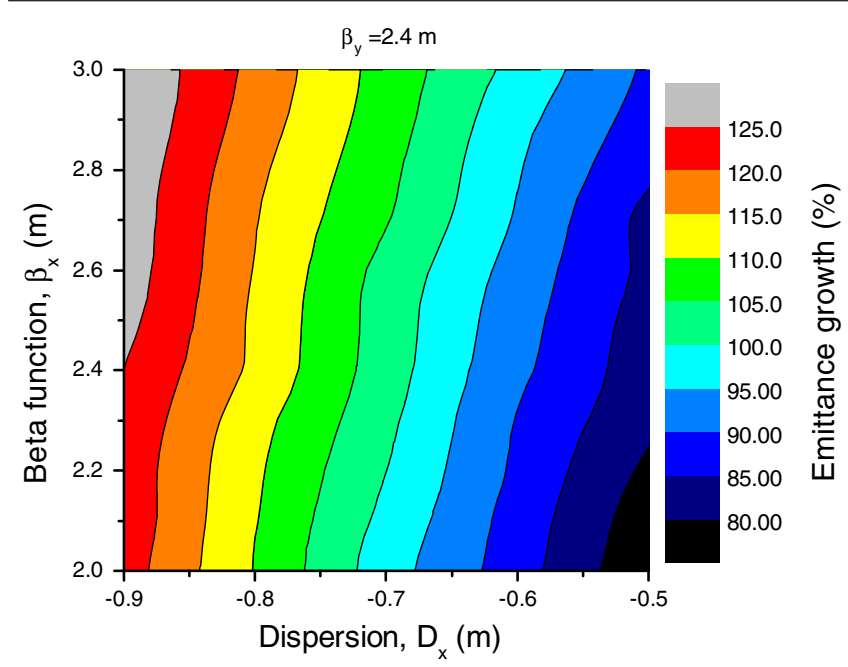

FIG. 7. Color map showing the corresponding horizontal emittance growth after the wedge as a function of the dispersion and beta function. The vertical scale on the right shows the percentage of emittance growth induced by the wedge compared to the no absorber case. All studies assume a single $\left(\mathrm{C}_{2} \mathrm{H}_{4}\right)_{n} 250 \mathrm{~mm}$ thick house-shaped wedge at a $162^{\circ}$ angle and assume the modified lattice $S_{2}$. The wedge is pointing along the horizontal direction with its apex placed near the beam center.

The transverse acceptance of the ring is limited by its aperture, which has a radius of $45 \mathrm{~mm}$ and betatron functions of $\beta_{x}=8 \mathrm{~m}$ and $\beta_{y}=18 \mathrm{~m}$. Accordingly, the acceptances that contain $95 \%$ of the beam are $A_{x}=$ $253 \mu \mathrm{m}$ and $A_{y}=113 \mu \mathrm{m}$, respectively. This could be rewritten as a rms emittance by dividing by 6 and obtaining $\varepsilon_{x} \approx 42 \mu \mathrm{m}$ and $\varepsilon_{y} \approx 19 \mu \mathrm{m}$, respectively. The transverse emittance increase induced by the wedge should be less than these aperture cuts. Figure 7 shows the horizontal emittance growth after the wedge for solution $S_{2}$ as a function of the beam optics after assuming a starting emittance of $12 \mu \mathrm{m}$ in both planes. The corresponding emittance increase in the vertical plane is $25 \%$. One can see that the growth is correlated to both dispersion and beta function. Most importantly, the growth is confined within the aforementioned storage ring acceptance boundaries which explains why the wedge has a positive impact on the stored muons.

From Fig. 6 we can see that when $D_{x}$ is small, we see a weak dependence with $\beta_{x}$. As the dispersion value increases, especially for $-D_{x}>0.3 \mathrm{~m}$, emittance exchange becomes strongly correlated to dispersion and transverse beta, meaning that both are key parameters that govern the performance of the wedge. Peak rates are achieved when $-D_{x}>1$ and $\beta_{x}$ are $<1 \mathrm{~m}$ where we see a notable increase on the number of stored muons. This fact is not surprising since more dispersion will imply a larger spread in momentum of the particles traversing the absorber while a smaller beta will ensure a reduced beam size and thus less emittance growth from scattering. For a practical point of view, however, achieving $\beta_{x}<1 \mathrm{~m}$ is not feasible in our present beam line. On the other hand, our numerical findings in Fig. 6 imply that even if we use Twiss parameters that are equivalent to either $S_{1}\left(\beta_{x}=2.4 \mathrm{~m}\right)$ or $S_{2}\left(\beta_{x}=2.7 \mathrm{~m}\right)$ we will still see a considerable gain within the $30 \%=-35 \%$ range.

\section{SIMULATED PERFORMANCE}

While our aforementioned Monte Carlo model provides a good first-order estimate on the emittance exchange performance, to further access the feasibility of using a wedge absorber in the Muon Campus, it must be studied under more realistic considerations. For instance, the present model is not providing any information on muon polarization which is one of the most critical parameters of the Muon g-2 Experiment. For this reason, we employ the tracking code G4beamline [26]. Using the Geant4 [27] package, the code incorporates key particle-matter physical processes (e.g., energy loss, straggling, multiple scattering) as well as includes muon decays and precession of the muon spin. In the simulation, virtual detectors where placed at various locations along the beam line. As a result, it was possible to evaluate the population of secondaries, their $6 \mathrm{D}$ phase space distribution, as well as the beam profiles over long distances. The code is also properly modified so that it fully incorporates all magnetic apertures according to the latest engineering specifications [1].

Figure 8 shows a schematic layout of the $13 \mathrm{~m}$ long horizontal bend string along the M5 line that is used in our G4beamline model. The yellow rectangular elements are dipoles, the cylindrical objects are quadrupoles, while the magenta colored elements, labeled as $W_{1}$ and $W_{2}$, depict the wedges. A more detailed illustration of the characteristics of the wedge is displayed in Fig. 9. For practical implementation, it is important to install such absorbers between existing lattice components with no modification to other nearby components. As a result, the available free space is limited to $130 \mathrm{~mm}$ [28]. Given this constraint and since the ideal thickness of a $\left(\mathrm{C}_{2} \mathrm{H}_{4}\right)_{n}$ wedge should be at least $220 \mathrm{~mm}$, we consider the inclusion of two equivalent wedges

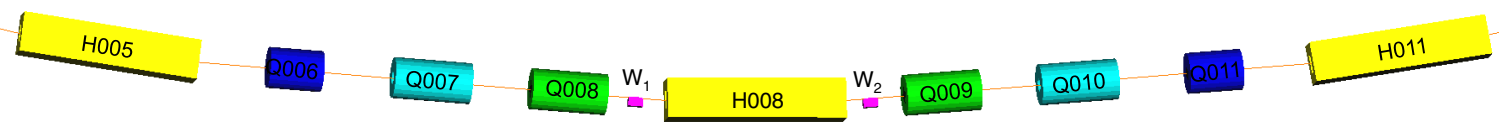

FIG. 8. Top view of the horizontal bend string along the M5 line. The yellow rectangular shaped elements are dipoles; the green, blue, and cyan cylinders are quadrupoles; and the magenta elements, which are labeled as $W_{1}$ and $W_{2}$, are the $\left(\mathrm{C}_{2} \mathrm{H}_{4}\right)_{n}$ absorbers. Both wedges are house shaped, $80 \mathrm{~mm}$ in height, $125 \mathrm{~mm}$ thick, and have an angle of $162^{\circ}$. All three dipoles are $1524 \mathrm{~mm}$ long. 


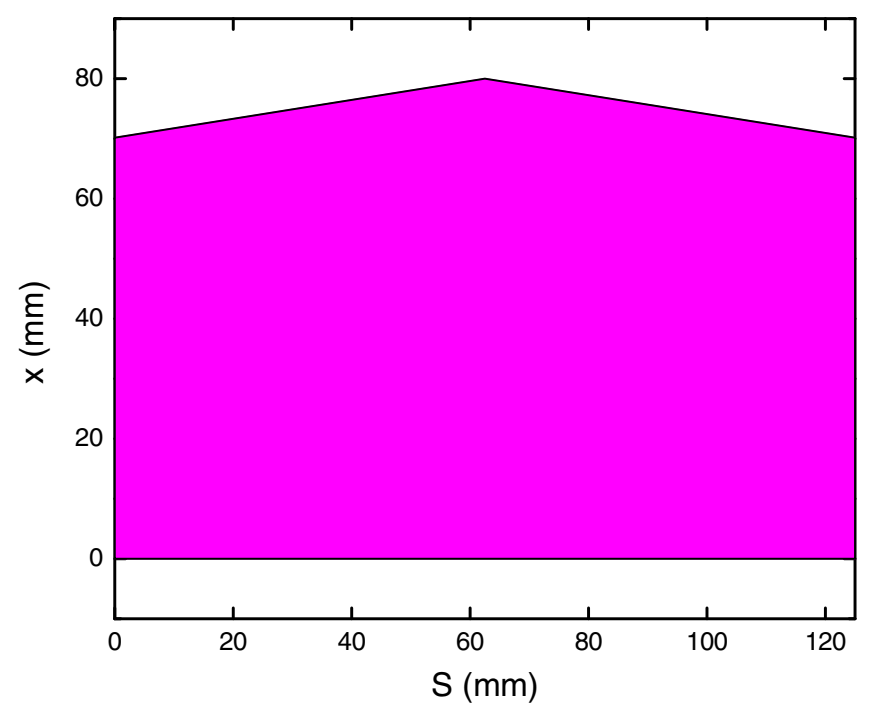

FIG. 9. Geometry of the simulated wedge. Note that both $W_{1}$ and $W_{2}$ wedges have the same dimensions.

with a maximum thickness of $125 \mathrm{~mm}$ each. Special care was taken in order to place them as close as possible to dipole H008. The reasoning becomes apparent after carefully examining the results in Fig. 3(b) and noting that its front edge is at $S=52.04 \mathrm{~m}$. Besides the fact that considerable dispersion exists, both $\beta_{x}$ and $\beta_{y}$ are close to their minimums near this region. Because scattering is highly correlated to the beta function, this implies the lowest possible emittance growth as well. We choose their center to be at the midpoint

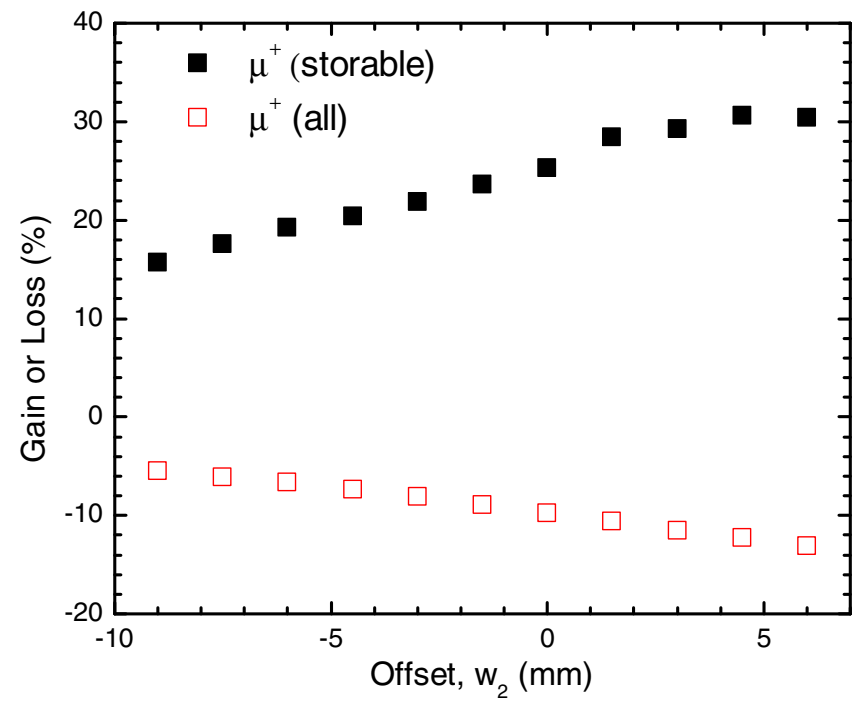

FIG. 10. Gain or losses of muons at the end of M5 compared to the case without a wedge as a function of the distance between the apex of $W_{2}$ and the beam line center. The red squares show the total intensity while the black squares show the storable muons only. As storable muons, we define muons that are contained within $0.15 \%$ of the central momentum. The simulation assumes two $\left(\mathrm{C}_{2} \mathrm{H}_{4}\right)_{n}$ absorbers with a thickness of $125 \mathrm{~mm}$ and a $162^{\circ}$ angle. The apex of $W_{1}$ is fixed at the beam line center. between $\mathrm{H} 008$ and the adjacent quadrupoles, i.e., at $S=$ $51.78 \mathrm{~m}$ (for $W_{1}$ ) and $S=53.82 \mathrm{~m}$ (for $W_{2}$ ). Unlike our studies in Sec. III, where a priori assumption of the initial beam distribution was made, we start upstream of $W_{1}$ by using the actual distribution that is the outcome of an end-toend simulation from the target [5]. Our simulation was initialized at the upstream edge of Extraction C-Magnet (ECMAG) at $S=0$ and for simplicity there was no readjustment of the beam line optics downstream the wedge from the original settings.

Figure 10 displays the beam intensity at the end of the M5 line for different wedge offsets using solution $S_{1}$. Note that as storable muons we define muons that are contained

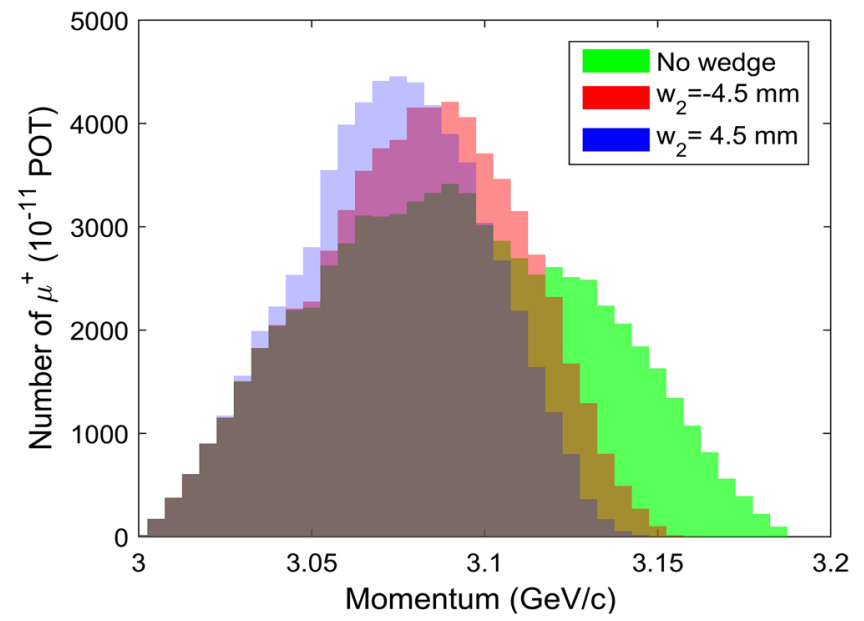

FIG. 11. Momentum distribution at the end of M5. With green we show the case without an absorber while with red and blue we show the distributions for different offsets of $W_{2}$. The apex of $W_{1}$ is fixed at the beam line center.

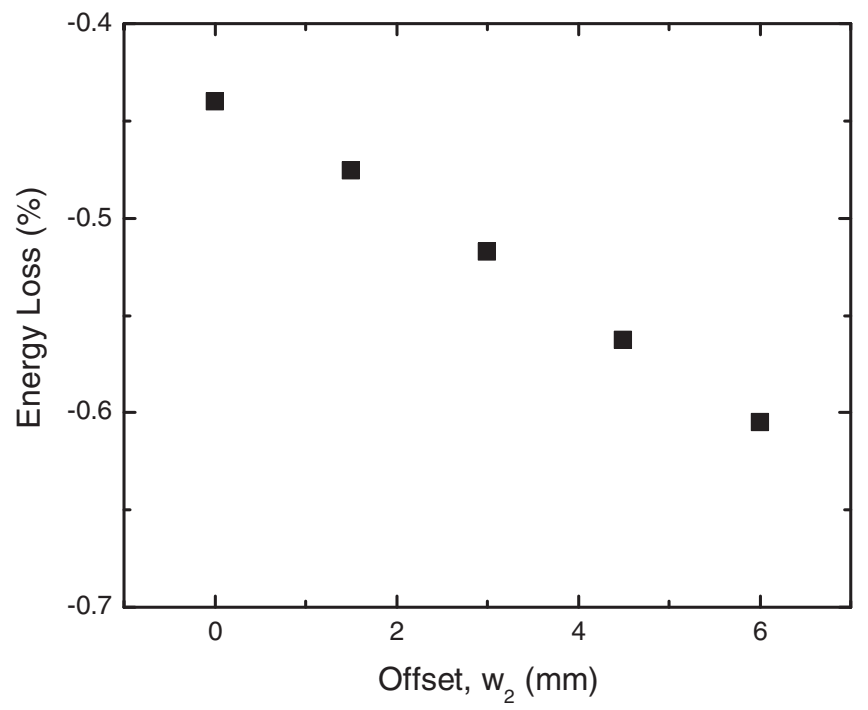

FIG. 12. Mean momentum variation of the muon distribution at the end of M5 as a function of the $W_{2}$ apex offset from the beam line center. The apex of $W_{1}$ is fixed at the beam line center. 
within $0.15 \%$ of $p_{m}$ and therefore have a high likelihood to survive as they pass into the ring downstream. For all simulations, we keep the apex of $W_{1}$ fixed at the beam line center $\left(w_{1}=0\right)$ so that it covers half of the beam, mainly the high-momentum particles, while we vary $w_{2}$ which is the distance between the apex of $W_{2}$ and the beam line center. Clearly, the total number of muons is strongly correlated to the amount of material seen by the beam, resulting in a steady decrease in intensity as $W_{2}$ is further inserted along the beam path. Conversely, the number of storable muons is reaching a plateau when $w_{2}=$ $3.0-6.0 \mathrm{~mm}$ providing evidence of an optimum operating point. The maximum gain occurs when $w_{2}=4.5 \mathrm{~mm}$ wherein the number of potentially stored muons has

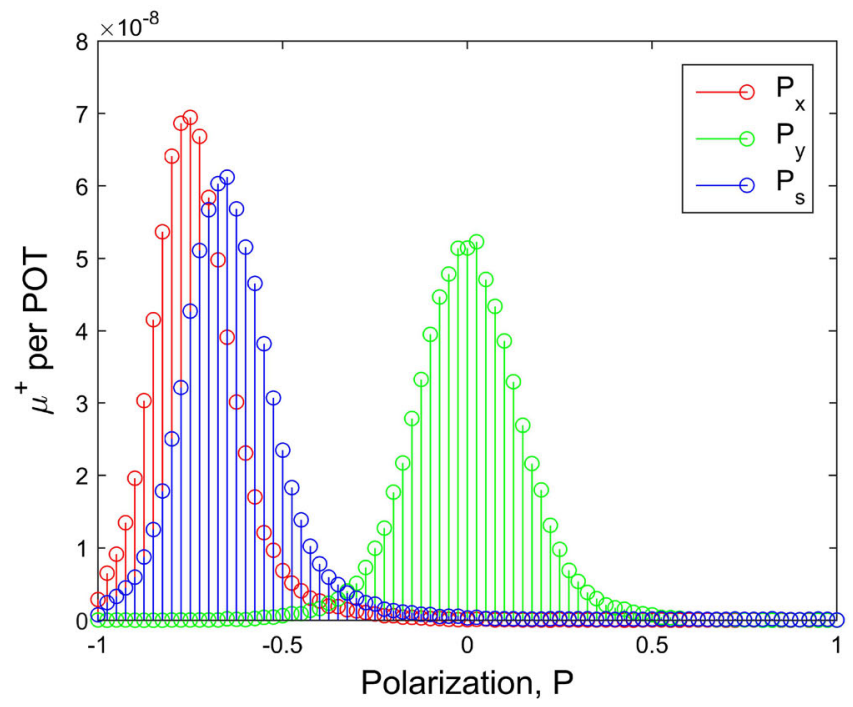

(a)

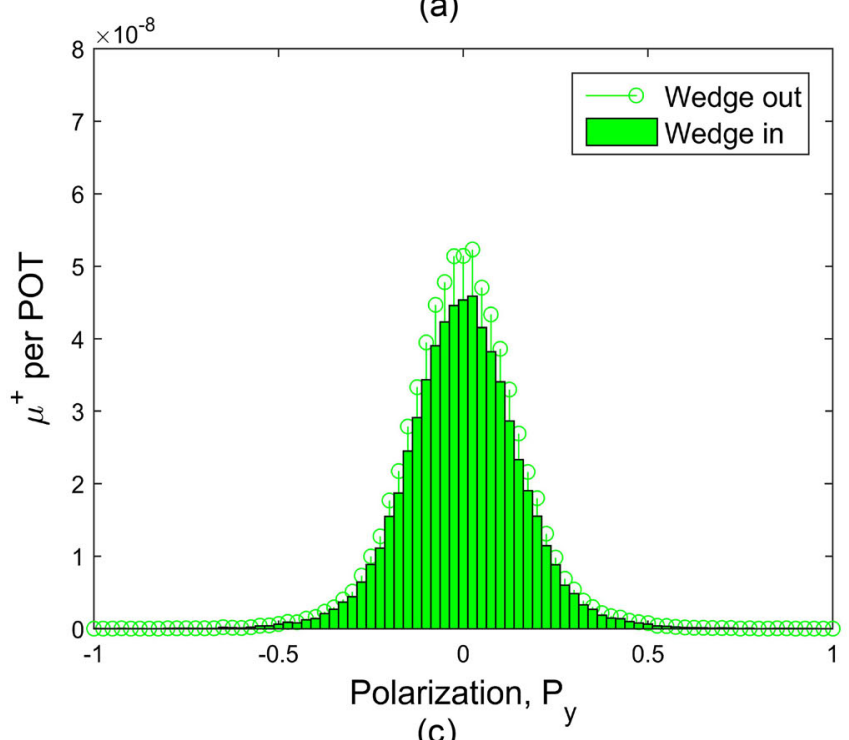

(c) increased by $31 \%$ while the total intensity has dropped by $13 \%$.

In our simulation, an unfortunate but not surprising finding was that the enhancement of potentially stored muons was accompanied by a substantial emittance growth that becomes more noticeable near $w_{2}=4.5 \mathrm{~mm}$. Quantitatively, we found that the beam reaching the end of M5 had a vertical emittance that was $25 \%$ higher compared to the one before the wedge, respectively. This is not unexpected, since emittance growth is primarily caused by multiple scattering on the absorber and is strongly correlated to the amount of material seen by the beam. On the other hand, the emittance growth can be suppressed by reducing the beta function so that better control of the

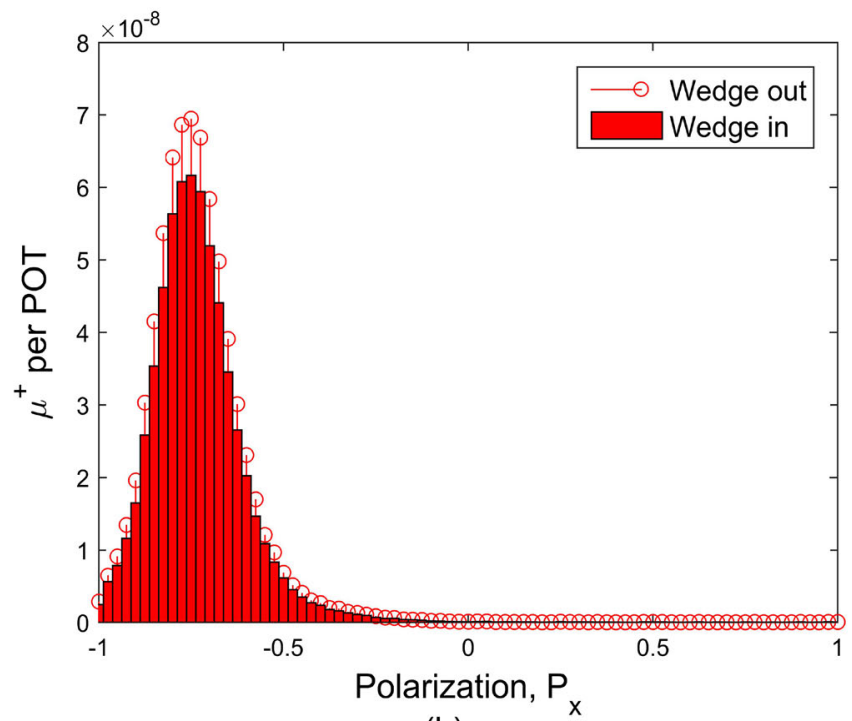

(b)

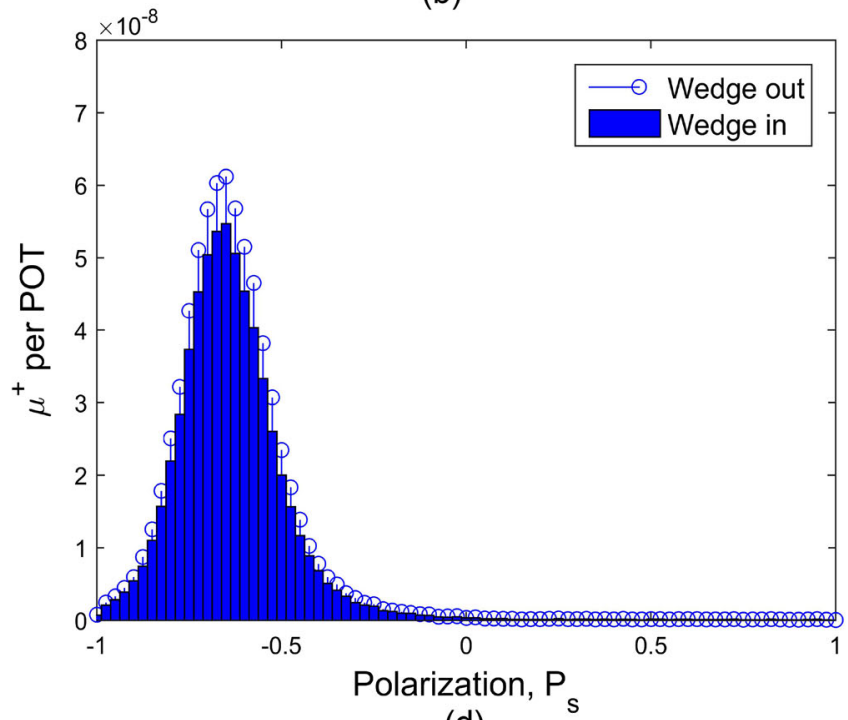

(d)

FIG. 13. Simulation results showing the distribution of the muon polarization at the end of M5. (a) Polarization without the wedge. (b) Polarization in the horizontal plane with and without wedge. (c) Polarization in the vertical plane with and without wedge. (d) Polarization in the longitudinal plane with and without wedge. In all cases the polarization remains unchanged when the absorber is inserted along the beam path. 
scattered particle trajectories within the wedge can be achieved. To confirm this hypothesis, we repeat the same simulation but this time using solution $S_{2}$. While we found the emittance growth in the horizontal plane to be almost the same as for $S_{1}$, the growth in the vertical plane was suppressed by a factor of 4 . In addition, it resulted in no perceptible difference in the number of storable muons compared to $S_{1}$.

Figure 11 displays the momentum distribution at the end of the M5 for different offsets of $W_{2}$. A salient feature of our study is that the low-momentum tail is not affected by the wedge, suggesting that the increase in storable muons is primarily caused from chopping off the high-momentum tail. Quantitatively, the original rms momentum spread $\sigma_{p} / p_{m}$ of $1.26 \%$ in the absence of an absorber, reduces to $0.86 \%$ when both wedges are inserted. Our simulation also captures well the dependence of the mean energy with wedge positioning. In particular Fig. 12 shows that if the apex of $W_{2}$ is placed at its optimum point, the mean energy decreases by $0.55 \%$ compared to the case without a wedge. This suggests that in order to enter the storage ring at the desired momentum of $3.1 \mathrm{GeV} / \mathrm{c}$, the beam preceding $W_{1}$ has to be at a slightly higher energy.

Next, we attempt to examine how the two absorbers impact the beam polarization. Based on the published parameters of Ref. [1], a reduction of the experimental uncertainty to $140 \mathrm{ppb}$ for the Muon g-2 Experiment will require a beam with a polarization $>90 \%$. Polarized muons are obtained from the weak decays of in flight pions: $\pi^{+} \rightarrow \mu^{+}+\nu_{\mu}$. Typically, the daughter muons have a very wide momentum spectrum in the lab frame that ranges from around one-half of the pion momentum (backward decays) to slightly greater than the pion momentum (forward decays), where forward and backward refers to the center-of-mass frame muon direction relative to the Lorentz boost between the frames. Since the momentum acceptance of the Muon Campus is relatively narrow i.e., $<2 \%$, it ensures that most muons are born from forwarddecayed pions, resulting in a highly polarized muon beam. Quantitatively, the simulation predicts that without the absorber the average beam polarization at the end of the M5 is 93\% [Fig. 13(a)] and almost equally shared between the horizontal and longitudinal planes, i.e., $\left\langle P_{x}\right\rangle=-0.729$ and $\left\langle P_{s}\right\rangle=-0.628$. If we now repeat the same simulation but with both $W_{1}$ and $W_{2}$ inserted, we find that the polarization remains unchanged in horizontal [Fig. 13(b)], vertical [Fig. 13(c)], and longitudinal planes [Fig. 13(d)]. Clearly, this indicates that the wedge has a negligible effect on polarization and therefore can be safely inserted along the beam path.

In order to further ascertain the potential benefits of a wedge absorber for the Muon g-2 Experiment, we track the above distributions over several turns inside the storage ring. We inject the beam with the same Twiss parameters every time, namely $\beta_{x}=2 \mathrm{~m}, a_{x}=-0.4, \beta_{y}=10 \mathrm{~m}$, and

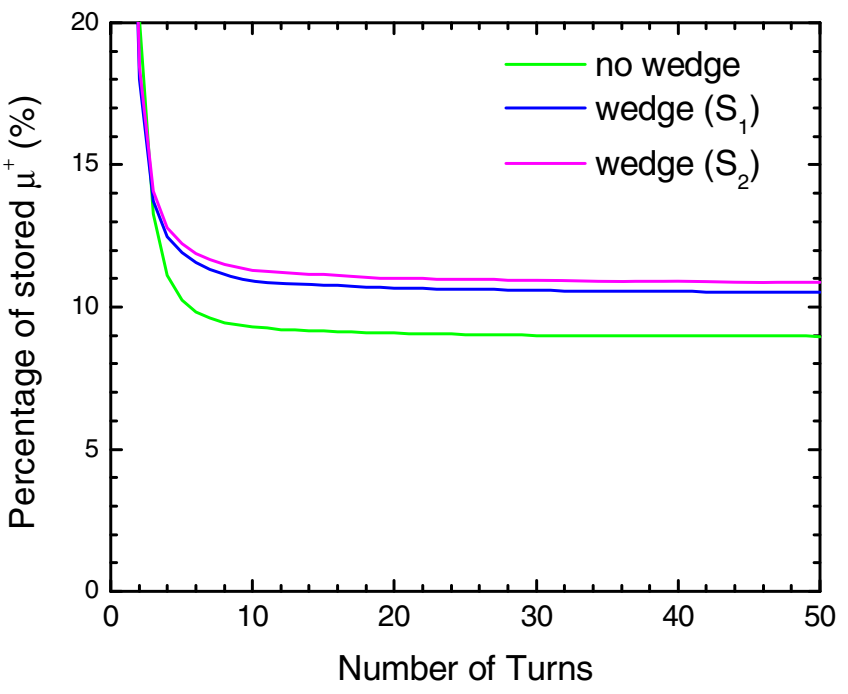

FIG. 14. Simulated transmission through the storage ring of the Muon g-2 Experiment. The green curve shows the case without the absorber. When the wedges are inserted, we see a gain in storable muons which depends on the nearby optics. The blue curve corresponds to optics solution $S_{1}$ while the magenta curve corresponds to optics solution $S_{2}$.

$a_{y}=0.2$. Based on the published parameters of Ref. [29], the aforementioned Twiss parameters will provide optimum capture efficiency in the ring. Figure 14 shows the transmission of muons as a function of the number of turns. In our study, we define capture efficiency as the ratio between the muons that survive 50 turns to the ones incident in the ring. Muons that hit apertures are considered lost and muon decay was not allowed. Clearly, one can see that when the wedges are inserted, both solutions see a considerable gain. Solution $\mathrm{S}_{2}$ achieves a gain $21 \%$ which is $4 \%$ more compared to $S_{1}$. This is not unexpected and can be understood as follows: The transverse acceptances of the ring that contain $95 \%$ of the beam are $A_{x}=253 \mu \mathrm{m}$ and $A_{y}=113 \mu \mathrm{m}$, respectively. This indicates that the emittance is the key parameter that governs the final transmission along the ring, especially in the vertical plane, since the acceptance is smaller. Since solution $S_{2}$ delivers a beam with a considerable smaller emittance in the vertical plane compared to $S_{1}$, it is expected to perform better and this fact is consistent with our present numerical findings. We conclude that the wedges have a positive impact on the Experiment as they can increase the storable beam intensity.

\section{SUMMARY}

In this paper we have discussed the design and performance of a wedge absorber system for momentum selection and momentum-spread reduction, potentially applicable to muon-based experiments. The concept relied on placing a wedge absorber at a point along a beam transport system with nonzero dispersion. The technique has direct relevance 
to precision-science experiments such as the Fermilab Muon g-2 Experiment. In particular, we showed that it can enhance the number of stored muons and therefore minimize the statistical uncertainty of the measurement. The absorber accomplished this by reducing the momentum spread of the beam via emittance exchange before injection into the storage ring. The improved statistics from delivery of more muons could push the capabilities of the Experiment into new regions of parameter space, allowing the measurement of the anomalous magnetic moment with unprecedented precision.

With the aid of this work, the needed material, its length, as well as the required lattice optics can now be specified to a level that a first-order cost and practicality study can be implemented. More specifically, our study revealed four interesting points. First, among several materials examined, polyethylene appeared the most promising choice based on cost and performance. Second, by taking into account the available space between magnetic elements, the ideal wedge-shaped block would have a maximum longitudinal thickness of about $125 \mathrm{~mm}$ with a tapered edge leading to a point at the beam center. The horizontal dimension of a wedge would be roughly $80 \mathrm{~mm}$ in order to cover the high-momentum beam part. Third, the wedge does not degrade the muon polarization, meaning that it remained $>90 \%$ as desired by the Muon g-2 Experiment. Fourth, simulations revealed that the addition of two such wedges in the beam line just upstream of the storage ring of the Muon g-2 Experiment could increase the number of stored muons at the $20 \%$ level with the available beam optics. As we showed, the potential for this number to increase further exists; however, it will require proper modification of the lattice parameters in the vicinity of the absorber.

\section{ACKNOWLEDGMENTS}

The authors are grateful to Joe Bradley, Nathan Froemming, James Morgan, William Morse, and David Neuffer for many discussions. Special thanks goes to Michael Syphers for the many useful discussions as well as for providing the theoretical input for the Monte Carlo code used in Sec. III. This research has been sponsored by the Laboratory Research and Development Program of Fermi National Accelerator Laboratory, managed by Fermi Research Alliance, LLC under Contract No. DEAC02-07CH11359 with the United States Department of Energy.

[1] J. Grange et al., arXiv:1501.06858.

[2] J. Mott, Nucl. Part. Phys. Proc. 287-288, 65 (2017).

[3] G.W. Bennett et al., Final report of the E821 muon anomalous magnetic moment measurement at BNL, Phys. Rev. D 73, 072003 (2006).

[4] G. Venanzoni, Nucl. Part. Phys. Proc. 273, 584 (2016).
[5] D. Stratakis, M. E. Convery, C. Johnstone, J. Johnstone, J. P. Morgan, D. Still, J. D. Crnkovic, V. Tishchenko, W. M. Morse, and M. J. Syphers, Accelerator performance analysis of the Fermilab Muon Campus, Phys. Rev. Accel. Beams 20, 111003 (2017).

[6] D. Tarazona, M. Berz, R. Hipple et al., Muon beam tracking and spin-orbit correlations for precision $\mathrm{g}-2$ measurements, Proceedings of IPAC 2016, Busan (JACow, Busan, Korea, 2016), p. 3397.

[7] D. Neuffer, Part. Accel. 14, 75 (1983).

[8] G. K. O'Neill, Storage-ring synchrotron: Device for highenergy physics research, Phys. Rev. 102, 1418 (1956).

[9] P. Snopok, G. Hanson, and A. Klier, Recent progress on the 6d cooling simulations in the guggenheim channel, Int. J. Mod. Phys. A 24, 987 (2009).

[10] J. Pasternak, Muon front end with a cooling ring, Nucl. Phys. B 149, 271 (2005).

[11] D. Stratakis, R. C. Fernow, J. S. Berg, and R. B. Palmer, Tapered channel for six-dimensional muon cooling towards micron-scale emittances, Phys. Rev. ST Accel. Beams 16, 091001 (2013).

[12] D. Stratakis, R. C. Fernow, J. S. Berg, and R. B. Palmer, Rectilinear six-dimensional ionization cooling channel for a muon collider: A theoretical and numerical study, Phys. Rev. ST Accel. Beams 18, 031003 (2015).

[13] D. Neuffer and A. V. Ginneken, Simulation studies of ionization cooling, Nucl. Instrum. Methods Phys. Res., Sect. A 403, 1 (1998).

[14] D. Stratakis, B. Drendel, J. P. Morgan, M. J. Syphers, and N. S. Froemming, Commissioning and first results of the Fermilab Muon Campus, Phys. Rev. Accel. Beams 22, 011001 (2019).

[15] J. Bradley et al., Initial studies into longitudinal ionization cooling for the Muon g-2 Experiment, Proceedings of IPAC 2018, Vancouver, BC, Canada (JACow, Vancouver, BC, Canada, 2018), p. 1522.

[16] M. D. Church and J. P. Marriner, The antiproton sources: Design and operation, Annu. Rev. Nucl. Part. Sci. 43, 253 (1993).

[17] http://mad.web.cern.ch/mad/ for information about the MAD program.

[18] D. L. Rubin et al., Muon Beam Dynamics and Spin Dynamics in the g-2 Storage Ring, Proceedings of IPAC 2018, Vancouver, BC, Canada (JACow, Vancouver, BC, Canada, 2018), p. 5029.

[19] N. Froemming et al., Commissioning the superconducting magnetic inflector system for the Muon g-2 Experiment, Proceedings of IPAC 2018, Vancouver, BC, Canada (JACow, Vancouver, BC, Canada, 2018), p. 1844.

[20] A. Yamamoto et al., The superconducting inflector for the BNL g-2 experiment, Nucl. Instrum. Methods Phys. Res., Sect. A 491, 23 (2002).

[21] A. N. Skrinsky and V. V. Parkhomchuk, Cooling methods for beams of charged particles, Sov. J. Part. Nucl. 12, 223 (1981).

[22] F. C. Fernow and J. C. Gallardo, Validity of the differential equations for ionization cooling, AIP Conf. Proc. 352, 170 (1996).

[23] M. J. Syphers, G Minus 2 Experiment Document 12020, 2018, https://gm2-docdb.fnal.gov/. 
[24] https://www.mathworks.com/ for information about the MATLAB mathematical computing software.

[25] J. Bradley, B. Drendel, and D. Stratakis, First measurement of traverse beam optics for the Fermilab Muon Campus using a magnet scanning technique, Nucl. Instrum. Methods Phys. Res., Sect. A 903, 32 (2018).

[26] http://www.muonsinternal.com/muons3/G4beamline for information about the G4beamline tracking simulation program.
[27] http://geant4.cern.ch/ for information about the Geant4 toolkit.

[28] J. Morgan (private communication).

[29] S. Kim, D. Rubin, D. Stratakis, and N. A. Froemming, The muon injection simulation study for the Muon g-2 experiment at Fermilab, Proceedings of NAPAC 2016, Chicago, IL (JAcow, Chicago, IL, USA, 2016), p. 803. 